\title{
Age affects the paracrine activity and differentiation potential of human adipose-derived stem cells
}

\author{
JEONG SEOP PARK ${ }^{1}$, GABEE PARK $^{2}$ and HYUN SOOK HONG ${ }^{1,3,4}$

\begin{abstract}
${ }^{1}$ Department of Biomedical Science and Technology, Graduate School, Kyung Hee University, Seoul 02447;
${ }^{2}$ Department of Genetic Engineering, College of Life Science and Graduate School of Biotechnology, Kyung Hee University, Yong In 17104; ${ }^{3}$ East-West Medical Research Institute, Kyung Hee University; ${ }^{4}$ Kyung Hee Institute of Regenerative Medicine (KIRM), Medical Science Research Institute, Kyung Hee University Hospital, Seoul 02447, Republic of Korea
\end{abstract}

Received September 1, 2020; Accepted December 3, 2020

DOI: $10.3892 / \mathrm{mmr} .2020 .11799$

\begin{abstract}
Stem cell therapy is considered a novel treatment modality for critical diseases. Adipose tissue is a rich and easily accessible source of stem cells. Adipose-derived stem cells (ADSCs) can be expanded ex vivo and possess characteristics similar to those derived from the bone marrow. However, the quality of ADSCs can be affected by age, underlying disease or the lifestyle of individuals. The aim of the present study was to explore the association between age and ADSC activity, including paracrine and differentiation potential. Adipose tissues from young (age <30 years) and elderly (age $>70$ years) groups were obtained, and ADSCs from each group were cultured in vitro. The effect of age on ADSC activity was investigated in vitro by evaluating the proliferation rate, adipo/osteogenic differentiation potential and cytokine profile using ELISA. The results revealed that increased age reduced cell activity and increased the doubling time of ADSCs, without causing profound morphological changes. The paracrine action of ADSCs was markedly altered by increased age, as demonstrated by reduced expression levels of vascular endothelial growth factor, stromal cell-derived factor- $1 \alpha$ and hepatocyte growth factor. Differentiation of ADSCs into osteoblasts or adipocytes rarely occurred in the elderly group compared with the young group. Overall, these results indicate
\end{abstract}

Correspondence to: Professor Hyun Sook Hong, Kyung Hee Institute of Regenerative Medicine (KIRM), Medical Science Research Institute, Kyung Hee University Hospital, Kyung Hee Daero 23, Seoul 02447, Republic of Korea

E-mail: hshong@khu.ac.kr

Abbreviations: ADSCs, adipose-derived stem cells; BMP-2, bone morphogenetic protein 2; BMPR1A, bone morphogenetic protein receptor 1; HGF, hepatocyte growth factor; SDF-1, stromal cell-derived factor-1; TGF- $\beta$, transforming growth factor- $\beta$; VEGF, vascular endothelial growth factor

Key words: adipose-derived stem cell, age, paracrine action, differentiation that age may affect the cellular function of ADSCs and should be considered prior to ADSC transplantation.

\section{Introduction}

Stem cell therapy has been broadly used to treat a variety of diseases, including acute/chronic inflammatory and vascular diseases (1-3). The primary sources of stem cells are the bone marrow (BM), dental tissue, adipose tissue, and umbilical cord blood. BM is considered the primary source of multipotent stem cells and thus has been used as an important source of stem cells for clinical cellular therapy (4-8). However, BM stem cell culture requires BM aspiration via femoral puncture, an invasive procedure that may be both a risk and a burden to patients. Additionally, BM stem cell viability ex vivo is influenced by the patient's unique background (9). Thus, BM stem cells from patients with critical illnesses may not be suitable for ex vivo culture for transplantation purposes.

Studies have been carried out to investigate adipose tissue as an alternative to BM, and adipose-derived stem cells (ADSCs) obtained through minimally invasive procedures have been found to express stem cell-specific markers. Additionally, these ADSCs have also been found to possess the ability for self-renewal, differentiation into multiple cell lineages, and paracrine action, which are similar characteristics to those observed in BM mesenchymal stem cells (BMSCs). Notably, ADSCs have a high rate of repopulation in vitro, thereby providing sufficient cell numbers for patient use; moreover, as compared to that of BMSCs, the cellular activity of ADSC has been reported to be rarely altered by the underlying disease condition of the donor. The efficacy of ADSCs has been confirmed in the treatment of many disorders and injuries, including muscle injury, cartilage damage, neurodegenerative disorders, and rheumatoid arthritis (10-13). Thus, ADSCs have emerged as a crucial alternative source of stem cells.

ADSC transplantation is typically performed using autologous stem cells from host adipose tissue; the main beneficiary group requiring stem cell therapy is the elderly, rather than the young. With aging, the number of inflammatory markers present in the human body also tends to increase $(14,15)$ and thus, the elderly are more susceptible to 
illnesses than the young. Alterations in the immune responsiveness of the elderly can affect their stem cell activity, given that stem cells are profoundly injured by changes in physiological conditions, such as aging or systemic inflammation. BMSCs from the elderly exhibit a reduced differentiation capacity with a low level of cytokine production (16-20). The same has been observed for ADSCs, and the reduced cell activity of ADSCs can undeniably lead to compromised therapeutic effects (21).

Therefore, donor age is a critical factor in estimating the efficacy of stem cell therapy. However, studies on the effect of age on ADSC activity remain relatively inconclusive; therefore, this study aimed to address this gap, which is crucial for stem cell therapy using ADSCs.

We hypothesized that age affects the cellular activity of ADSCs. To verify our hypothesis, we comparatively analyzed the essential functions of ADSCs obtained from young and elderly donors, by evaluating the cell proliferation rate, differentiation potential, and cytokine profile.

\section{Materials and methods}

Materials. Healthy adipose tissues were provided by the Kyung Hee University Medical Center [Total of eight donors (6 male, 2 female); Seoul, Korea; (IRB\# 2016-12-022)] with donors' written consent. Adipose tissue $(2 \times 2 \mathrm{~cm})$ was suspended in saline in a $50 \mathrm{ml}$ tube at $4^{\circ} \mathrm{C}$ and transferred to the cell culture room. ADSC isolation was performed immediately upon arrival to the culture room. ADSCs from healthy young individuals were purchased from ScienCell Research Laboratories (individuals $<30$ years old). Alizarin Red S powder, Oil Red O solution, formaldehyde solution, and cetylpyridinium chloride were purchased from Sigma-Aldrich; Merck KGaA. Stempro osteogenesis and adipogenesis kits, $\alpha$-MEM medium, and FBS were purchased from Gibco; Thermo Fisher Scientific, Inc. Phosphate-buffered saline, penicillin/streptomycin, and $0.25 \%$ trypsin-EDTA solution were provided by Welgene. Vascular endothelial growth factor (VEGF), hepatocyte grow factor (HGF), stromal cell-derived factor- $\alpha$ (SDF- $1 \alpha)$, transforming growth factor- $\beta 1$ (TGF- $\beta 1$ ), and the bone morphogenetic protein (BMP)-2 ELISA kits were obtained from R\&D Systems.

Cell culture. ADSCs were isolated as previously reported (21) and cultured in an $\alpha$-MEM medium supplemented with $10 \%$ fetal bovine serum (FBS) and $1 \%$ penicillin/streptomycin. The culture medium was replaced with fresh medium every other day. The ADSCs between 3rd and 5th passages were used for all the experiments. Endothelial progenitor cells (EPCs) were cultured from BM mononuclear cells (STEMCELL, 70001.4) in an EGM-2 medium (Lonza) on fibronectin-coated dishes. The medium was changed every other day and cells in passage 2 were used for this study. ADSC was analyzed for specific marker expression by FACSCalibur using Cell Quest software (version 3.0, BD Biosciences).

WST-1 assay. The ADSCs $\left(2 \times 10^{4}\right.$ cells/well) were seeded in a 96-well plate and incubated for $24 \mathrm{~h}$. Ten microliters of WST-1 solution was added at $10 \%$ of the total medium volume, and the plate was incubated for $90 \mathrm{~min}$ at $37^{\circ} \mathrm{C}$ with $5 \% \mathrm{CO}_{2}$. Optical density values for each well were measured at $450 \mathrm{~nm}$ using a Versa Max Microplate Reader (Molecular Devices).
Cytokine measurement. The ADSCs $\left(5 \times 10^{5}\right.$ cells/well) were seeded in a 6 -well plate in $2.5 \mathrm{ml}$ of $\alpha$-MEM medium. The concentrations of VEGF, HGF, SDF- $1 \alpha$, TGF- $\beta 1$ and BMP-2 in the conditioned medium were analyzed using ELISA kits according to the manufacturer's instructions.

Matrigel tube formation assay. Matrigel (BD Biosciences, $100 \mu \mathrm{l}$ ) was applied on 48 well-plates and incubated for $30 \mathrm{~min}$ at $37^{\circ} \mathrm{C}$. The EPCs $\left(2 \times 10^{4}\right.$ cells at passage 2$)$ in conditioned medium from ADSCs (young or elderly) were added to the Matrigel matrix and incubated for $3 \mathrm{~h}$ at $37^{\circ} \mathrm{C}$ with $5 \% \mathrm{CO}_{2}$. The effect of ADSC paracrine factors on tube structure was observed under a stereomicroscope.

Sample preparation and western blot analysis. Protein lysates were quantified and then denatured and electrophoresed using sodium dodecyl sulfate-polyacrylamide gel electrophoresis and transferred to a nitrocellulose membrane. After blocking with $5 \%$ skim milk, the membranes were incubated with primary antibodies for bone morphogenetic protein receptor $1 \mathrm{~A}$ (BMPR1A) and GAPDH, followed by anti-immunoglobulin G horseradish peroxidase-conjugated secondary antibody. The blots were developed and visualized with ECL (Dogen Bio) and analyzed under chemiluminescence using Amersham imager 600 (GE Healthcare). Protein expression levels were quantified using the ImageJ software.

Osteogenic induction assay. The ADSCs were maintained in $\alpha$-MEM medium. Osteogenic medium was replaced with the fresh medium every three days. At day 20, the cells were fixed using 3.7\% formaldehyde and stained with Alizarin Red S solution $(2 \%, \mathrm{w} / \mathrm{w})$ for $10 \mathrm{~min}$ prior to visualization of calcium deposition. Alizarin Red S was eluted using cetylpyridinium chloride $(10 \%, \mathrm{w} / \mathrm{v})$, and calcium deposition was quantified as the absorbance value at $560 \mathrm{~nm}$ (Molecular Devices).

Adipogenic induction assay. Adipogenesis was induced using the Stempro adipogenesis kit following the manufacturer's instructions. At day 15, the cells were fixed using 3.7\% formaldehyde and stained with Oil Red $\mathrm{O}$ solution to evaluate lipid droplet formation. Oil Red $\mathrm{O}$ was eluted using isopropyl alcohol (Daejung, Siheung, Korea), and the absorbance value was measured at $490 \mathrm{~nm}$ (Molecular Devices).

Statistical analysis. All data are presented as mean \pm standard deviation (SD) of three independent experiments. Statistical analyses of all data were carried out using an unpaired, two-tailed Student's t-test. P-values $<0.05$ were interpreted to indicate statistically significant differences $(\mathrm{P}<0.05, \mathrm{P}<0.01$, $\mathrm{P}<0.001)$.

\section{Results}

Age reduces the viability and proliferation rate of ADSCs. The ADSCs harvested from the young ( $<30$ years) and elderly ( $>70$ years) groups were cultured in vitro and analyzed. Cellular morphology was not affected by age (Fig. 1A). ADSCs exhibited a typical shape of fibroblastic cells that sustained over a long period in vitro. Specific marker gene expression was also not affected by age (Table SI). However, ADSC activity varied 
considerably depending on age. The viability of ADSCs was significantly reduced in the elderly group as compared to that in the young group (Fig. 1B). This reduction in cell activity also led to an increase in population doubling time (Fig. 1C).

Stem cell transplantation requires a large number of cells, approximately $1 \times 10^{8}-1 \times 10^{9}$, indicating that the ADSCs from the elderly require more time to reach the desired cell number for transplantation, as compared to those from the young. An increase in the time required for cellular expansion is a key indicator of cellular senescence or dysfunction. Specifically, ADSC viability can be affected by the physiological condition of the elderly and may rapidly undergo cellular senescence during ex vivo culture. Therefore, ADSCs from the elderly may lose their therapeutic efficacy during ex vivo culture.

Paracrine action of ADSCs is altered by age. Paracrine actions of stem cells account for the hallmark function of stem cell therapy in vivo. Stem cells tend to lose their ability to secret cytokines or growth factors due to senescence. Given that transplanted stem cells primarily act through paracrine factors, preservation of their ability to produce cytokines can be critical in predicting their treatment efficacy following transplantation (14).

For screening secreted cytokines, conditioned medium from ADSCs was analyzed with cytokine array (Fig. S1). Compared to the ADSCs from the young group, those from the old group tended to produce higher levels of inflammatory factors and lower levels of angiogenic factors, including HGF and VEGF.

Among the identified secretory factors in ADSCs, we quantitatively determined the secretion levels of angiogenic factors using ELISA. VEGF, HGF, SDF- $1 \alpha$ and TGF- $\beta$ are involved in vascularization or bone formation, as well as cell survival. These represent important factors to consider in stem cell transplantation for the treatment of vascular or skeletal diseases (22-25).

Levels of VEGF, HGF, and SDF-1 $\alpha$ were clearly reduced in the ADSCs from the elderly group when compared to those from the young group (Fig. 2A-C; VEGF, Young: 6.23 \pm 0.23 , Elderly: $5.09 \pm 0.36 \mathrm{pg} / \mathrm{ml} / \mu \mathrm{g}$ protein, $\mathrm{P}<0.001$; HGF Young: 18.22 \pm 0.41 , Elderly: $3.04 \pm 0.15 \mathrm{pg} / \mathrm{ml} / \mu$ g protein, $\mathrm{P}<0.001$; SDF-1 $\alpha$ Young: $7.5 \pm 0.41$, Elderly: $4.1 \pm 1.18 \mathrm{pg} / \mathrm{ml} / \mu \mathrm{g}$ protein, $\mathrm{P}<0.01)$. However, TGF- $\beta$ production was elevated in the ADSCs from the elderly group (Fig. 2D; TGF- $\beta$, Young: $8.57 \pm 0.61$, Elderly: $11.71 \pm 0.58 \mathrm{pg} / \mathrm{ml} / \mu \mathrm{g}$ protein, $\mathrm{P}<0.001)$.

The difference in the levels of angiogenic factors produced by ADSCs of the young and elderly groups was expected to affect angiogenesis in vivo. To determine the effect of soluble factors from ADSCs on vessel formation, conditioned medium from ADSCs was collected and then applied to EPCs on matrigel in vitro (Fig. 2E). As shown in Fig. 2F, the conditioned medium from ADSCs from the young group could promote tube formation by EPCs much more than that from the ADSCs from the elderly group.

These data suggest that age can alter the cytokine secretion profile of ADSCs and this may be related to treatment efficacy in vivo.

Age weakens the differentiation potential of ADSCs. We have proven that age can influence cell repopulation rate and cytokine secretion. This suggests that age may disrupt the
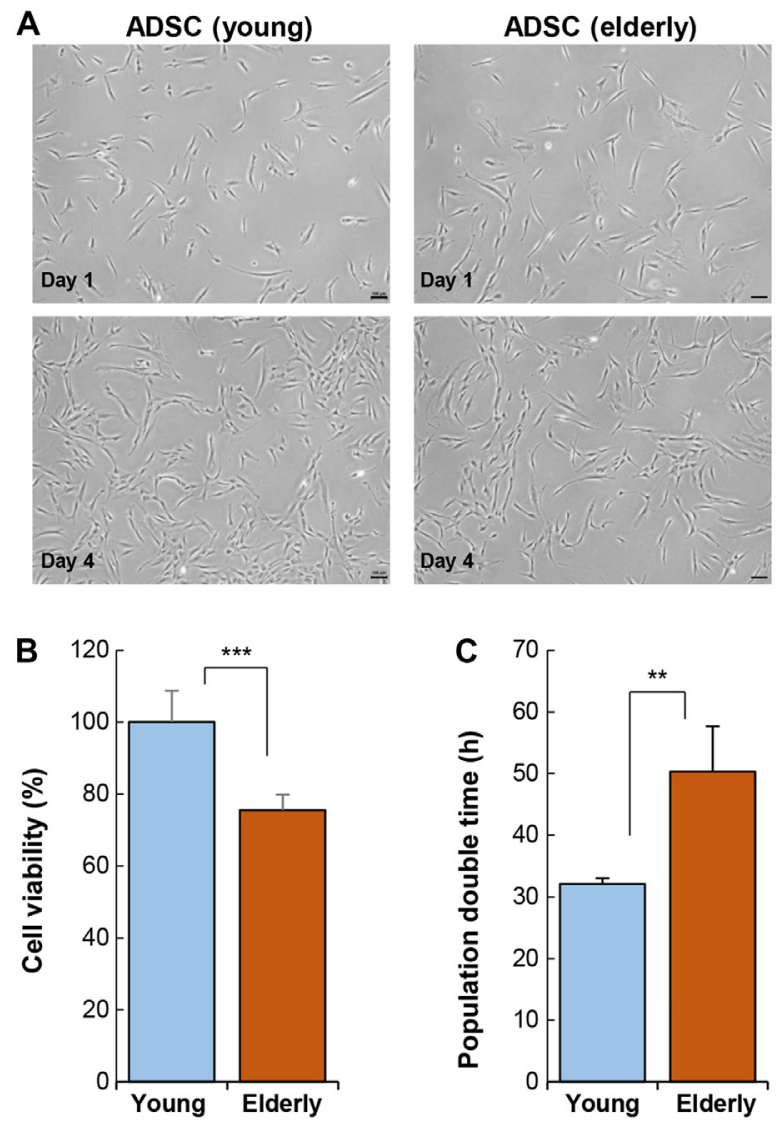

Figure 1. Effect of age on the cellular activity of ADSCs. (A) ADSCs were isolated from adipose tissue from the young and elderly and cultured in vitro. Light microscopy images of cell morphology. Cellular morphology was compared using light microscopy images. Scale bar, $100 \mu \mathrm{m}$. (B) A water soluble tetrazolium salts-1 assay was performed to compare cellular activity of ADSCs. (C) Population doubling time of ADSCs. Data are presented as the mean $\pm \mathrm{SD}$ of at least three replicates for each group. ${ }^{* *} \mathrm{P}<0.01$ and ${ }^{* * * *} \mathrm{P}<0.001$. ADSCs, adipose-derived stem cells.

differentiation potential of ADSCs. To test this, we induced adipogenesis and osteogenesis in ADSCs from the young and elderly groups and compared their differentiation potential.

Osteogenic induction of ADSCs was carried out for 20 days and calcium deposition was then assessed using Alizarin Red S staining (Fig. 3A and B). This corroborated the impairment of the osteogenic potential of ADSCs from the elderly group.

Osteogenesis is driven by BMP-2 signaling, emphasizing the role of BMP signaling in osteogenesis (26). The level of BMP-2 secreted from ADSCs was not influenced by age (Fig. 3C). Next, the expression of BMP receptor 1A (BMPR1A; receptor for BMP-2) was determined, revealing that the ADSCs from the elderly group have reduced BMPR1A expression as compared to that of the ADSCs from the young group (Fig. 3C). This suggests that ADSCs from the elderly might have a weak response to exogenous BMP-2 due to poor expression of its receptor, which can contribute to poor osteogenesis.

In addition to osteogenesis, we also verified the adipogenic potential of ADSCs by culturing the ADSCs in adipogenic media for two weeks and then staining cellular lipid droplets using Oil Red O (Fig. 4). ADSCs from the elderly group showed a significant reduction in adipogenic potential compared to that of the young group. 
A

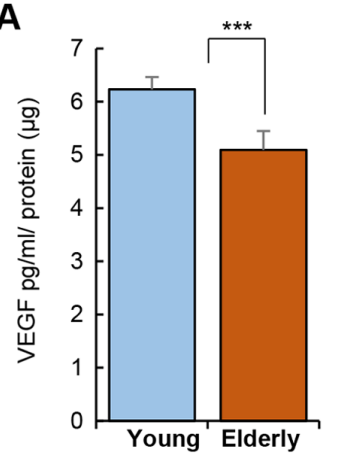

B

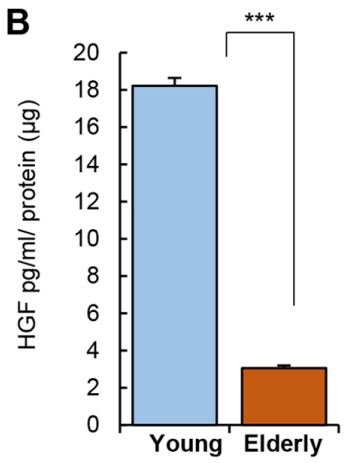

C

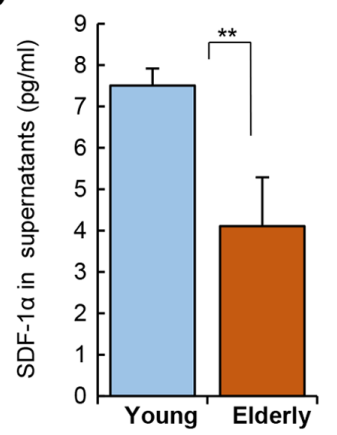

D

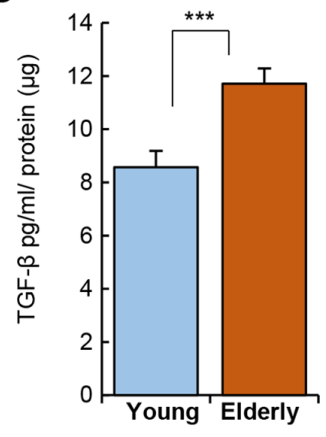

E
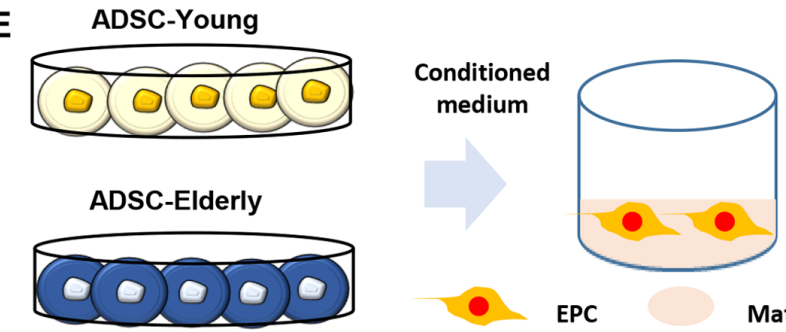

$37^{\circ} \mathrm{C}, 3 \mathrm{~h}$

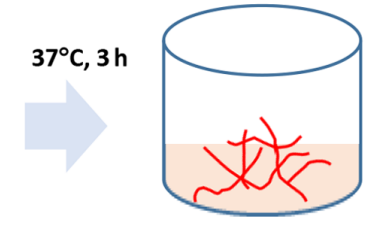

EPC

Matrigel

$\mathbf{F}$
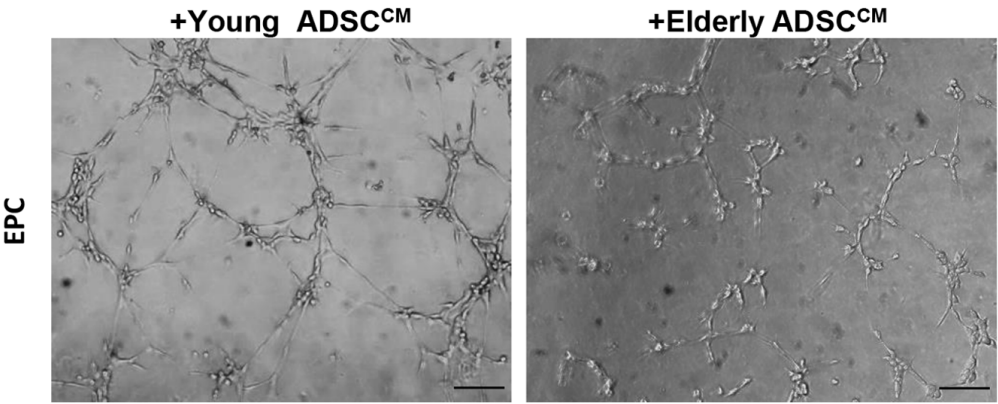

Figure 2. Effect of age on the paracrine potential of ADSCs. ADSCs were cultured and the culture medium was collected for the quantification of cytokines Quantification for (A) VEGF, (B) HGF, (C) SDF-1 $\alpha$ and (D) TGF- $\beta$ was performed using ELISA. (E) Experimental scheme for Matrigel assay with EPC. Conditioned medium of ADSC was treated with EPCs on Matrigel. (F) Representative images for tube formation. Data are presented as the mean \pm SD of at least three replicates for each group. Scale bar, $100 \mu \mathrm{m} .{ }^{* *} \mathrm{P}<0.01$ and ${ }^{* * *} \mathrm{P}<0.001$. ADSCs, adipose-derived stem cells; HGF, hepatocyte growth factor; SDF-1 $\alpha$, stromal cell-derived factor- $\alpha$; TGF- $\beta$, transforming growth factor- $\beta$; VEGF, vascular endothelial growth factor; EPC, endothelial progenitor cell.

Collectively, the differentiation potential of ADSCs from the elderly group was clearly impaired, regardless of the specific cell type polarization. This overall depletion in the differentiation potential of ADSCs in the elderly group may be caused by alterations in paracrine action or receptor expression, given that cell differentiation is induced by specific growth factors.

\section{Discussion}

Stem cell therapy remains a revolutionary and novel treatment modality with considerable potential to become one of the most important breakthroughs of medical science $(19,27)$. The global stem cell therapy market is highly competitive, and growth in this market is further fueled by the increasing number of scientific research activities utilizing the therapeutic potency of stem cells for medical treatment.

BMSCs are the most actively utilized cell type for transplantation, and their therapeutic effect on various chronic diseases is evidenced by treatment outcomes, such as suppression of inflammation or enhancement of tissue growth to promote regeneration. However, depending on the underlying disease condition of a given patient, BMSCs may not be viable due to the risk of access or poor cell viability. Adipose tissue has been actively investigated as a promising treatment for incurable disorders (10-13). ADSC therapy exhibits therapeutic effects on neurodegenerative disorders, cardiac injuries, and cutaneous damage. Thus, ADSCs are undoubtedly a novel clinical therapeutic agent. To validate the efficacy of ADSC therapy, the cellular function of ADSCs should be analyzed prior to transplantation, and this standard should be considered as a quality control.

This study explored the effect of age on the cellular activity of ADSCs. Although ADSCs have inherent self-renewal capacity and exhibit paracrine action in vivo, these functions were expected to be altered by the donor's age. Previous reports have explored the relationship between aging and ADSC activity (28-31). However, these studies mainly focused on the effect of aging on the efficacy and differentiation of stem cells in non-clinical settings and determined the expression 
A
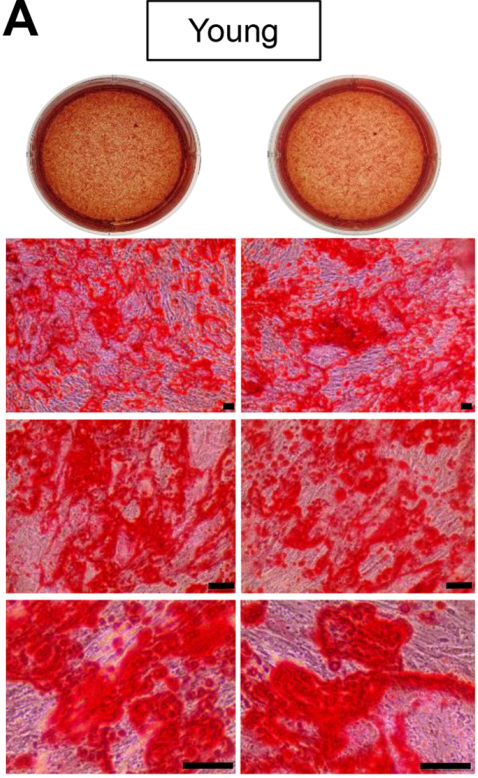

C

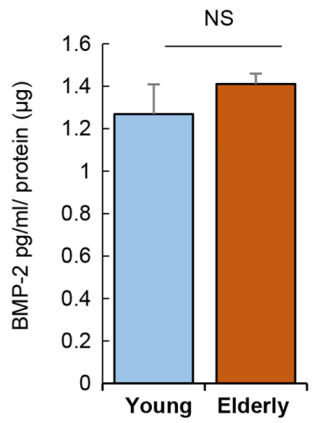

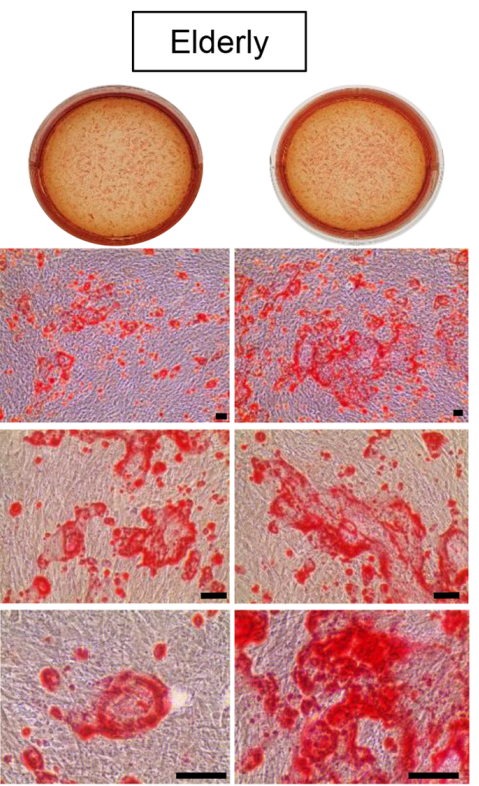

B

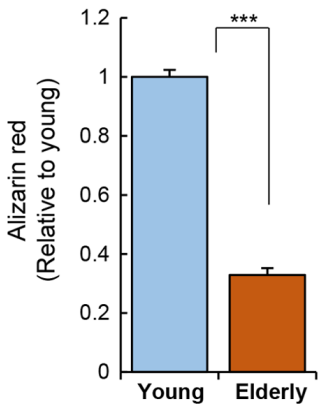

D

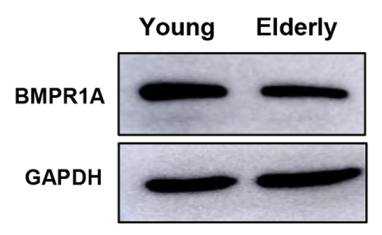

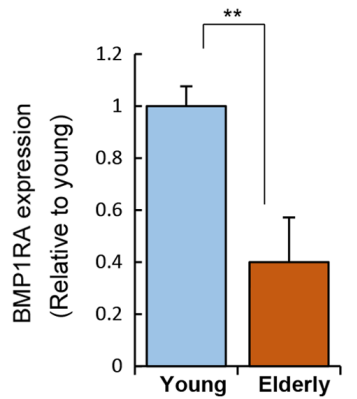

Figure 3. Effect of age on the osteogenic differentiation potential of ADSCs. (A) Osteogenic induction was performed for 20 days. ADSCs from the young or elderly were seeded, and the medium was replaced with fresh medium every 3 days. ADSCs were fixed after osteogenic induction and stained using Alizarin red S. Calcium deposition was observed as red color of Alizarin red S solution. Scale bar, $100 \mu \mathrm{m}$. (B) Alizarin red was quantified. (C) Measurement of BMP-2 in conditioned medium was carried out using an ELISA. (D) Expression levels of BMPR1A in ADSCs were determined by western blotting. Data are presented as the mean $\pm \mathrm{SD}$ of at least three replicate wells for each group. ${ }^{* * *} \mathrm{P}<0.01$ and ${ }^{* * * *} \mathrm{P}<0.001$. ADSCs, adipose-derived stem cells; BMP-2, bone morphogenetic protein 2; NS, not significant; BMPR1A, bone morphogenetic protein receptor 1A.

A

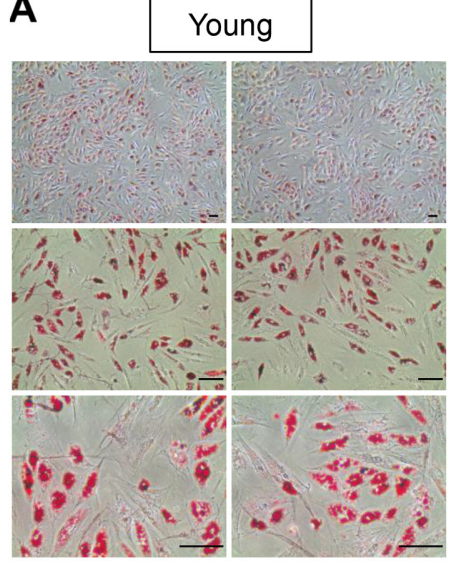

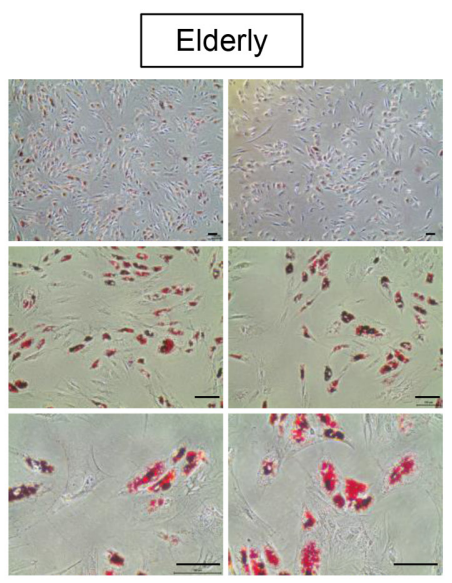

B

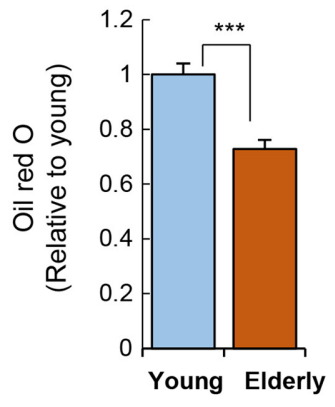

Figure 4. Effect of age on the adipogenic differentiation potential of ADSCs. (A) Adipogenic induction of ADSCs was performed over a period of 15 days. ADSCs were seeded at a density of $5 \times 10^{5}$ cells/well, and the medium was replaced with a fresh medium every 3 days. Following induction, cells were fixed and stained using Oil Red O. Scale bar, $100 \mu \mathrm{m}$. (B) Quantification of adipocytes stained with Oil Red O. Data are presented as the mean \pm SD of at least three replicates for each group. ${ }^{* * *} \mathrm{P}<0.001$. ADSCs, adipose-derived stem cells.

of paracrine factors at the transcriptional level. Although this work provided important insights, it was limited.
In this study, ADSCs from the elderly group showed low activity and repopulation rate compared to those from the 


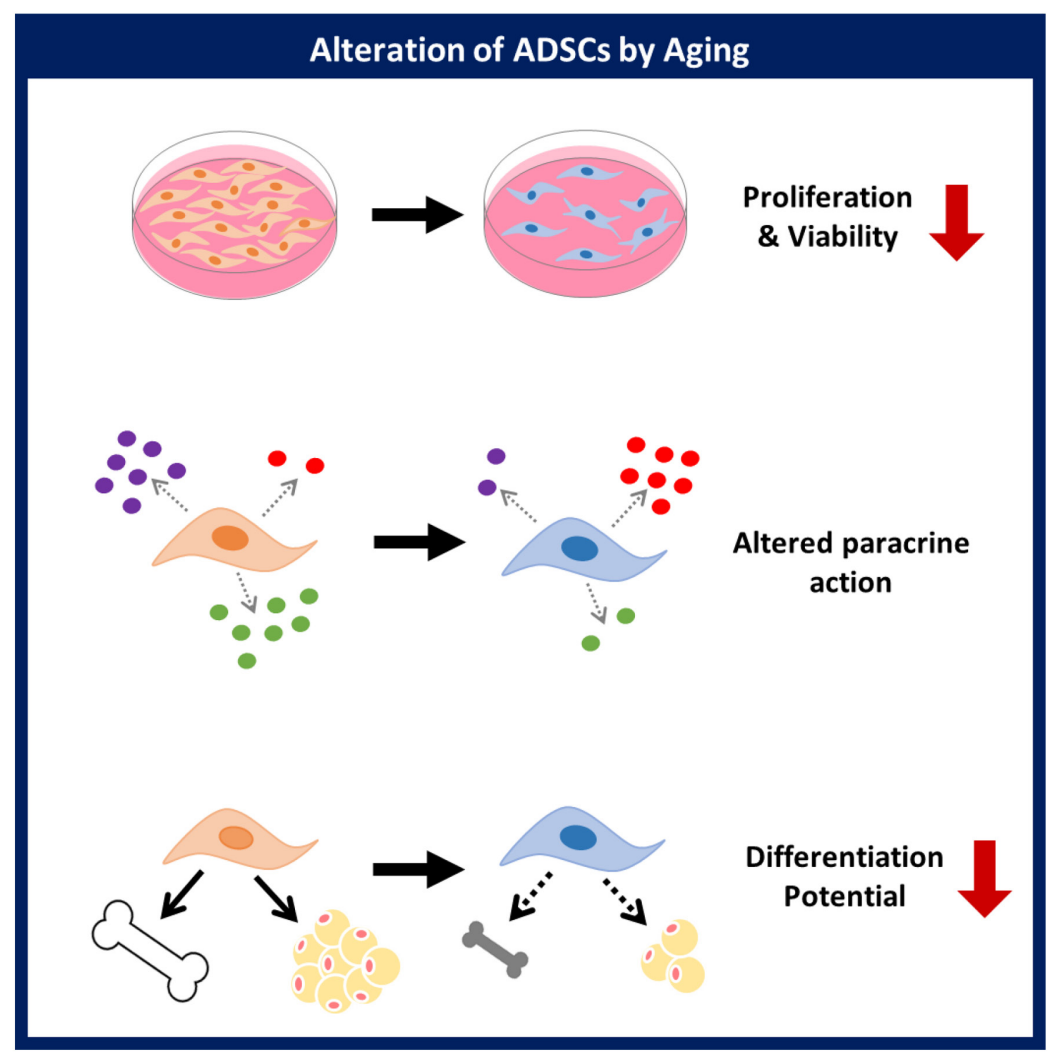

Figure 5. Summary of the effect of age on ADSC function. Aging induces alteration of viability, paracrine action and differentiation potential of ADSCs. ADSCs, adipose-derived stem cells.

young group. The levels of cytokine secretion were also quite different in the young group compared to those in the elderly. Notably, the production of angiogenic factors, including VEGF, SDF- $1 \alpha$, and HGF, was reduced in the ADSCs from the elderly group. These differences indicate that stem cell therapy using ADSCs from the elderly may not provide the expected paracrine action and cannot survive in vivo.

This low repopulation rate and weakened paracrine potential may lead to the impairment of the differentiation potential of stem cells. We found that ADSCs from the elderly group had a poor differentiation potential when polarized into osteoblasts or adipocytes, whereas ADSCs from the young group showed a prompt response to differentiation stimuli. This difference might be attributed to the altered expression of ligands/receptors in ADSCs due to aging.

Aging is a complex physiological process in the human body that encompasses a wide spectrum of biological processes, including oxidative stress, chronic inflammation, and cellular senescence. This complex physiological environment can affect stem cell viability. From a clinical perspective, ADSC therapy is often used in older patients. Many previous trials have relentlessly aimed to improve the stem cell proliferation rate to achieve the desired cell number for transplantation purposes, but have overlooked the aspects of paracrine action or differentiation capacity. ADSCs from the elderly with chronic inflammation were found to secrete higher levels of pro-inflammatory cytokines compared to those from the young. Particularly, the ADSCs with altered cytokine profiles may predispose the recipients to certain risks and thus ADSC activity should be examined in the elderly, prior to transplantation.
Taken together, with the above-mentioned information on the relationship between age and the cellular activity of ADSCs prior to transplantation, we can prepare additional procedures to augment the function of ADSCs derived from elderly patients.

Aging is accompanied by chronic diseases and stem cell therapy is chiefly applied in aged patients; therefore, we could not ignore the effect of diseases in age-mediated stem cell impairment. As such, the study of the complex response from elderly patients may be practically meaningful in clinical settings.

This study demonstrated that a donor's age affects the proliferative activity, paracrine action, and differentiation potential of ADSCs. Evaluation of the cellular activity of ADSCs based on age will be helpful for the development of ADSCs as a cellular therapeutic agent in stem cell therapy (Fig. 5). The relationship between specific background diseases and stem cell activity will be explored in future studies.

\section{Acknowledgements}

Not applicable.

\section{Funding}

The present study was supported by a Korean Health Technology R\&D Project grant (grant no. HI18C1492) from the Ministry of Health and Welfare (Sejong, South Korea) and Basic Science Research Program through the National Research Foundation of Korea (NRF) funded by the Ministry of Education (grant no. 2018R1D1A1B0704104813). 


\section{Availability of data and materials}

The datasets used and/or analyzed during the current study are available from the corresponding author on reasonable request.

\section{Authors' contributions}

HSH conceived and designed the study. JSP, HSH and GP analyzed and interpreted the data. JSP and HSH wrote the article. JSP, HSH and GP gave final approval of the article. All authors read and approved the final manuscript.

\section{Ethics approval and consent to participate}

All experimental procedures were performed in accordance with the ethical standards as recommended by Kyung Hee University Hospital. Donor samples were obtained with written consent after personal explanation (IRB\# 2016-12-022; Institutional Review Board of Kyung Hee University Hospital, Seoul, Republic of Korea).

\section{Patient consent for publication}

Not applicable.

\section{Competing interests}

The authors declare that they have no competing interests.

\section{References}

1. Baraniak PR and McDevitt TC: Stem cell paracrine actions and tissue regeneration. Regen Med 5: 121-143, 2010.

2. Biehl JK and Russell B: Introduction to stem cell therapy. J Cardiovasc Nurs 24: 98-105, 2009.

3. Goradel NH, Hour FG, Negahdari B, Malekshahi ZV, Hashemzehi M, Masoudifar A and Mirzaei H: Stem cell therapy: A new therapeutic option for cardiovascular diseases. J Cell Biochem 119: 95-104, 2018

4. Chen L, Tredget EE, Wu PY and Wu Y: Paracrine factors of mesenchymal stem cells recruit macrophages and endothelial lineage cells and enhance wound healing. PLoS One 2: e1886, 2008.

5. Uemura R, Xu M, Ahmad N and Ashraf M: Bone marrow stem cells prevent left ventricular remodeling of ischemic heart through paracrine signaling. Circ Res 9: 1414-1421, 2006.

6. Miao C, Lei M, Hu W, Han S and Wang Q: A brief review: The therapeutic potential of bone marrow mesenchymal stem cells in myocardial infarction. Stem Cell Res Ther 8: 242, 2017.

7. Xu S, Zhou Z, Li H, Liu Z, Pan X, Wang F, Huang Y, Li X, Xiao Y, Pan J, et al: BMSCs ameliorate septic coagulopathy by suppressing inflammation in cecal ligation and puncture-induced sepsis. J Cell Sci 131: jcs211151, 2018.

8. Watanabe S, Uchida K, Nakajima H, Matsuo H, Sugita D, Yoshida A, Honjoh K, Johnson WE and Baba H: Early transplantation of mesenchymal stem cells after spinal cord injury relieves pain hypersensitivity through suppression of pain-related signaling cascades and reduced inflammatory cell recruitment. Stem Cells 33: 1902-1914, 2015.

9. Wang Q, Zhao B, Li C, Rong JS, Tao SQ and Tao TZ: Decreased proliferation ability and differentiation potential of mesenchymal stem cells of osteoporosis rat. Asian Pac J Trop Med 7: 358-363, 2014.

10. Kesireddy V: Evaluation of adipose-derived stem cells for tissue-engineered muscle repair construct-mediated repair of a murine model of volumetric muscle loss injury. Int Nanomedicine 11: 1461-1473, 2016.
11. Kasir R, Vernekar VN and Laurencin CT: Regenerative engineering of cartilage using adipose-derived stem cells. Regen Eng Transl Med 1: 42-49, 2015.

12. Chi K, Fu RH, Huang YC, Chen SY, Hsu CJ, Lin SZ, Tu CT, Chang LH, Wu PA and Liu SP: Adipose-derived stem cells stimulated with n-butylidenephthalide exhibit therapeutic effects in a mouse model of Parkinson's disease. Cell Transplant 27: 456-470, 2018

13. Ueyama H, Okano T, Orita K, Mamoto K, Sobajima S, Iwaguro H and Nakamura H: Local transplantation of adipose-derived stem cells has a significant therapeutic effect in a mouse model of rheumatoid arthritis. Sci Rep 10: 3076, 2020.

14. Bektas A, Schurman SH, Sen R and Ferrucci L: Aging, inflammation and the environment. Exp Gerontol 105: 10-18, 2018.

15. Ferrucci L and Fabbri E: Inflammageing: Chronic inflammation in ageing, cardiovascular disease, and frailty. Nat Rev Cardiol 15: 505-522, 2018.

16. Asumda FZ and Chase PB: Age-related changes in rat bone-marrow mesenchymal stem cell plasticity. BMC Cell Biol 12: 44, 2011

17. Baek SM, Son Y and Hong HS: Substance P blocks the impairment of paracrine potential of MSC due to long term culture. Mol Cell Toxicol 14: 283-290, 2018.

18. Stolzing A, Jones E, McGonagle D and Scutt A: Age-related changes in human bone marrow-derived mesenchymal stem cells: consequences for cell therapies. Mech Ageing Dev 129: 163-173, 2008.

19. Sun LY, Zhang HY, Feng XB, Hou YY, Lu LW and Fan LM: Abnormality of bone marrow-derived mesenchymal stem cells in patients with systemic lupus erythematosus. Lupus 16: 121-128, 2007.

20. Murphy JM, Dixon K, Beck S, Fabian D, Feldman A and Barry F: Reduced chondrogenic and adipogenic activity of mesenchymal stem cells from patients with advanced osteoarthritis. Arthritis Rheum 46: 704-713, 2002.

21. Kim S, Piao J, Son Y and Hong HS: Substance P enhances proliferation and paracrine potential of adipose-derived stem cells in vitro. Biochem Biophys Res Commun 485: 131-137, 2017.

22. $\mathrm{Hu} \mathrm{K}$ and Olsen BR: The roles of vascular endothelial growth factor in bone repair and regeneration. Bone 91: 30-38, 2016.

23. Zhen R, Yang J, Wang Y, Li Y, Chen B, Song Y, Ma G and Yang B: Hepatocyte growth factor improves bone regeneration via the bone morphogenetic protein-2-mediated NF- $\mathrm{kB}$ signaling pathway. Mol Med Rep 17: 6045-6053, 2018.

24. Li Y, Chang S, Li W, Tang G, Ma Y, Liu Y, Yuan F, Zhang Z, Yang GY and Wang Y: cxcl12-engineered endothelial progenitor cells enhance neurogenesis and angiogenesis after ischemic brain injury in mice. Stem Cell Res Ther 9: 139, 2018.

25. Chen G, Deng C and Li YP: TGF- $\beta$ and BMP signaling in osteoblast differentiation and bone formation. Int J Biol Sci 8 272-288, 2012.

26. Salazar VS, Gamer LW and Rosen V: BMP signalling in skeletal development, disease and repair. Nat Rev Endocrinol 12: 203-221, 2016

27. Zakrzewski W, Dobrzyński M, Szymonowicz M and Rybak Z: Stem cell: Past, present, and future. Stem Cell Res Ther 10: 68, 2019.

28. Dufrane D: Impact of age on human adipose stem cells for bone tissue engineering. Cell Transplant 26: 1496-1504, 2017.

29. Wei W, Niklason L and Steinbacher DM: The effect of age on human adipose-derived stem cells. Plast Reconstr Surg 131: 27-37, 2013

30. Jin Y, Yang L, Zhang Y, Gao W, Yao Z, Song Y and Wang Y: Effects of age on biological and functional characterization of adipose-derived stem cells from patients with end-stage liver disease. Mol Med Rep 16: 3510-3518, 2017.

31. Marędziak M, Marycz K, Tomaszewski K, Kornicka K and Henry B: The influence of aging on the regenerative potential of human adipose derived mesenchymal stem cells. Stem Cells Int 2016: 2152435, 2016

This work is licensed under a Creative Commons Attribution-NonCommercial-NoDerivatives 4.0 International (CC BY-NC-ND 4.0) License. 